Editorial

\title{
Special issue of Microelectronics Journal on the Conference on Design of Circuits and Integrated Systems 2011 (DCIS 2011)
}

This special issue of the Microeletronics Journal is dedicated to the 26th edition of the Conference on Design of Circuits and Integrated Systems (dcis.org) which took place in Albufeira, Portugal, on 16-18 November, 2011. The participants in the DCIS series of conferences bring together a wealth of knowledge and experience, and have collectively witnessed and contributed to the amazing progress of today's microelectronics and microsystems products and technologies. They built-up a dedicated community, that, year after year, looks forward to meeting and participating in lively technical discussions.

From the 80 technical papers presented at the conference, 16 were selected to go through an additional reviewing process from which the group of 12 papers included in this special issue came out. In this process each paper was reviewed by at least three new experts. The topics addressed in these papers provide a good picture of the conference scope, embracing design and test of integrated circuits and systems, technologies, and applications.

Communications is one of the domains that have deserved the attention of DCIS participants. The papers "Multigigabit Analog Equalizers for Plastic Optical Fibers" by Cecilia Gimeno et al. and "Frequency-to-Digital Conversion Based on a Sampled PhaseLocked Loop" by Francisco Colodro et al. address the design of CMOS analog and mixed-signal circuits for, respectively, continuous-time equalizers aiming at compensating the low bandwidth of plastic optical fibers, and a new architecture for frequency to digital conversion based on a PLL FM demodulator with simplified analog content. Communication systems design involves trade-offs among performance, power consumption, reliability and cost. These papers address power consumption by means of low-voltage operation and use of digital circuits to minimize static power consumption.

Power efficiency is also addressed by the paper "Improved Power Efficiency for DVB-SH Transmitters", where Jose G. Doblado et al. propose an optimization of the active constellation extension algorithm in digital video broadcasting-satellite services to handheld (DVB_SH) systems as a means to reduce both transmitters' peak to average power ratio and cubic metric figures. Reduction of power levels, namely, to reduce distortion, may compromise transmission error rates. Improved low-density parity-check short/medium codes can be used to improve OFDM modulation allowing for better performance at the cost of a small increase of computational effort as described by José Gerald in "A Fast LDPC Encoder/Decoder for Small/Medium Codes".
Power consumption and process, voltage and temperature (PVT) variations are critical issues for the design of analog circuits in deep submicron MOS technologies, as discussed in the papers "Design Tradeoffs for Sub-mW CMOS Biomedical Limiting Amplifiers" by Javier Ramos et al., "Comparative Study on Time-to-Digital Converters for Low-Power RFID Tag Sensors" by Ainara Jimenez-Irastorza et al., "Design of Micropower Class AB Transconductors: A Systematic Approach" by Antonio J. Lopez-Martin et al. and "A $90 \mu \mathrm{m} \times 64 \mu \mathrm{m}$ $225 \mu \mathrm{W}$ Class-AB CMOS Differential Flipped Voltage Follower with Output Driving Capability up to 100 pF" by Carlos Muñiz-Montero et al. In addition to a proper selection of topologies, that are adapted to the targeted application field, the approaches employed include the elimination of the need for common-mode feedback blocks to control the output common-mode voltage, adoption of digital topologies instead of their analog counterparts, and the use of functional reconfiguration and built-in calibration schemes.

New applications requiring radiation detectors have appeared over the last decade. In "Readout Schemes for Low Noise SinglePhoton Avalanche Diodes Fabricated in Conventional HV-CMOS Technologies" three different pixels based on single-photon avalanche diodes operating in gated mode are presented by Eva Vilella et al., while Alejandro Garzon Camacho et al. describe in "Design and Experimental Results of a Preamplifier for Particles Tracking in Secondary Electron Detectors" a preamplifier for the front-end block of low-pressure gaseous secondary electron detectors, which shows better performance compared to previously reported devices.

Moving on to the technology integration level, the paper "Closed-Form Expressions for the Coupling Capacitance of Metal Fill Tiles in VLSI Circuits" by Nikolaos A. Tsatsoulis et al. derives a closed-form formula for the computation of the capacitive coupling introduced by dummy metal tiles placed in areas left empty of metallization.

Finally, Santiago Elvira et al. propose an improved indoor localization and orientation system in "ALO: an Ultrasound System for Localization and Orientation Based on Angles". The system is based on the detection of reception angles of the ultrasonic signal after the application of a differential time of arrival technique, which exhibits reduced computational cost, and is thus suitable for hardware implementation in field-programmable gate arrays.

José Machado da Silva, Sylvie Renaud, João Canas Ferreira 